\title{
Fibroscan como diagnóstico de hipertensión portal en pacientes cirróticos
}

Laura Perez ORCID: 0000-0003-0258-8410 Bachiller Medicina. Facultad de Medicina. UdelaR.

Valentina Pincelli ORCID: 0000-0002-2375-509X Bachiller Medicina. Facultad de Medicina. UdelaR.

Lorena Pittamiglio ORCID: 0000-0002-9068-7322 Bachiller Medicina. Facultad de Medicina. UdelaR.

Mateo Rodríguez Pezzino ORCID: 0000-0003-3147-6004

Bachiller Medicina. Facultad de Medicina. UdelaR.

Damián Santos ORCID: 0000-0002-6363-0558 Bachiller Medicina. Facultad de Medicina. UdelaR.

Micaela Saralegui ORCID: 0000-0003-1263-4871 Bachiller Medicina. Facultad de Medicina. UdelaR.

Gastón Nieto ORCID: 0000-0001-5420-1893 Internista. Hepatólogo. Asistente Clínica Médica. UdelaR.

Mercedes Perendones

ORCID: 0000-0002-3846-2396 Internista. Hepatólogo. Profesor Agregado Clínica Médica. UdelaR.

Marcelo Chiarella ORCID: 0000-0002-9715-4553 Internista. Profesor Adjunto Clínica Médica. UdelaR.

\section{Fibroscan as a diagnosis of portal hypertension in cirrhotic patients Fibroscan como diagnóstico de hipertensão portal em pacientes cirróticos}

Resumen: Introducción: La hipertensión portal es un síndrome frecuente en las hepatopatías crónicas. Entre sus etiologías destaca la cirrosis hepática, responsable en la gran mayoría de los casos. Desde la incorporación de la Elastografía de Transición (Fibroscan) en Uruguay, no se han realizado estudios a nivel nacional que relacionen los resultados obtenidos mediante esta técnica con la presencia de hipertensión portal en pacientes cirróticos. Objetivo: Caracterizar la población cirrótica diagnosticada mediante Fibroscan, atendida en la policlínica de Hepatología del Hospital Pasteur. Material y Métodos: Se estudiaron los pacientes con diagnóstico de cirrosis hepática de cualquier etiología asistidos y controlados en la policlínica de Hepatología del Hospital Pasteur en el periodo de 2015 a 2018. Resultados: Se incluyeron 49 pacientes, de los cuales 34 presentaban hipertensión portal. Se encontró mayor prevalencia de cirrosis hepática en hombres con etiología alcohólica y por infección por virus de la hepatitis C. Se halló asociación entre valores de ET $\geq 15 \mathrm{kPa}$ y la presencia de hipertensión portal. No fue posible demostrar asociación estadísticamente significativa entre valores de ET $\geq 15 \mathrm{kPa}$ y la presencia de várices esófago gástricas y/o gastropatía por hipertensión portal. Conclusiones: El punto de corte utilizado en el Fibroscan es útil para diagnóstico de hipertensión portal. Es necesario continuar realizando fibrogastroscopía para el diagnóstico de la misma.

Palabras clave: Hipertensión portal, elastografía de transición (Fibroscan), cirrosis hepática, várices esófago-gástricas.

Abstract: Introduction: Portal hypertension is a syndrome that is frequently present in chronic liver diseases. Within the causes that results in portal hypertension, liver cirrhosis outstands, being responsible for most cases. Since the incorporation of transient elastography (Fibroscan) in Uruguay, no research has been conducted at a national level that correlates the results obtained by Fibroscan with the presence of portal hypertension in cirrhotic patients. Objectives: To characterize cirrhotic population diagnosed by Fibroscan who are assisted in the Hepatology polyclinic of Hospital Pasteur. Material and Methods: Patients with diagnosis of liver cirrhosis of any etiology were studied, who have been assisted and monitored in the Hepatology polyclinic of Hospital Pasteur in the period 2015 - 2018. Results: 49 patients were included, of which 34 presented elements of portal hypertension. A higher prevalence of cirrhosis was found in men with alcoholic etiology and infection with hepatitis $\mathrm{C}$ virus. An association was found between Fibroscan values $\geq 15 \mathrm{kPa}$ and the presence of portal hypertension. It was not possible to demonstrate a statistically significant association between Fibroscan values $\geq 15 \mathrm{kPa}$ and the presence of gastric esophageal varices and/or gastropathy due to portal hypertension. Conclusions: The cut-off point used in Fibroscan is useful for the diagnosis of portal hypertension. It is necessary to continue performing fibrogastroscopy for the diagnosis of portal hypertension. 
Resumo: Introdução: A hipertensão portal é uma síndrome comum nas doenças hepáticas crônicas. Dentre suas etiologias, destaca-se a cirrose hepática, responsável na grande maioria dos casos. Desde a incorporação da Elastografia de Transição (Fibroscan) no Uruguai, não foram realizados estudos nacionais que relacionem os resultados obtidos por essa técnica com a presença de PHT em pacientes cirróticos. Objetivo: Caracterizar a população cirrótica diagnosticada por Fibroscan atendida na Policlínica de Hepatologia do Hospital Pasteur. Material e Métodos: Foram estudados pacientes com diagnóstico de cirrose hepática de qualquer etiologia assistida e controlada na Policlínica de Hepatologia do Hospital Pasteur no período de 2015 a 2018. Resultados: Foram incluídos no estudo 49 pacientes, dos quais 34 apresentavam elementos de hipertensão portal foi encontrada maior prevalência de cirrose hepática em homens com etiologia alcoólica e infecção pelo vírus da hepatite C. Foi encontrada associação entre valores de Fibroscan $\geq 15 \mathrm{kPa}$ e presença de hipertensão portal. Não foi possível demonstrar associação estatisticamente significante entre valores de Fibroscan $\geq$ $15 \mathrm{kPa}$ e presença de varizes esofágicas gástricas e / ou gastropatia por hipertensão portal. Conclusões: $O$ ponto de corte utilizado no Fibroscan é útil para o diagnóstico da hipertensão portal. É necessário continuar realizando fibrogastroscopia para o diagnóstico de hipertensão portal.

Palavras-chave: Hipertensão portal, elastografia de transição, cirrose hepática, varizes esofágicogástricas. 


\section{Introducción}

La hipertensión portal (HTP) es un síndrome que se caracteriza por el aumento de la presión hidrostática en el interior del sistema venoso portal, definido por un incremento del gradiente de presión entre la vena porta y la vena cava por encima de $5 \mathrm{mmHg}$.(1) Valores de presión portal $>5$ $\mathrm{mmHg}$ y $<10 \mathrm{mmHg}$ representan un estado subclínico de HTP; y valores $>10 \mathrm{mmHg}$ determinan una HTP clínicamente significativa.(2) Este síndrome determina la formación de colaterales portosistémicas, formando anastomosis profundas y superficiales. ${ }^{(3)}$ Las varices gastro-esofágicas (VGE) están presentes en el 50\% de los pacientes cirróticos, siendo éstas las derivaciones porto-sistémicas más relevantes clínicamente. ${ }^{(4)}$.

La valoración tardía de la presencia de HTP en los pacientes portadores de cirrosis implica un deterioro en la calidad de vida de los mismos, una elevada morbi-mortalidad, y un alto costo sanitario.

\section{Existen diversos métodos diagnósticos para valorar la presión portal.}

El goldstandard es a través de la determinación del gradiente de presión venosa hepática (GPVH), un método diagnóstico directo e invasivo que requiere de hospitalización, no estando exento de riesgos y complicaciones, por lo que no se realiza de forma rutinaria.

Existen parámetros clínicos que ponen de manifiesto la HTP como son la aparición de complicaciones que la misma ocasiona como son la presencia de encefalopatía hepática, hemorragia digestiva por várices esófago-gástricas, ascitis; y parámetros paraclínicos hematimétricos como la disminución del recuento plaquetario o un gradiente de albúmina suero-ascitis $($ GASA $) \geq 1,1 \mathrm{gr} / \mathrm{dl}^{(1)}$

Los estudios imagenológicos abdominales como la ecografía doppler, la tomografía y la resonancia nuclear magnética, pueden ayudar al diagnóstico de HTP, al constatar la presencia de elementos indirectos como son la existencia de recanalización del ligamento redondo, desarrollo de circulación intra-abdominal (hepato-renal, hepato-mesentérica, etc), presencia de esplenomegalia, permitiendo definir las características anatómicas de la vena porta; sin embargo no son útiles para determinar la HTP subclínica ni cuantificar la extensión de la HTP.

Por este motivo se han buscado nuevos métodos no invasivos para aproximar el diagnóstico de HTP. (2)

En los últimos años, surge como método diagnóstico la elastografía de transición (ET)

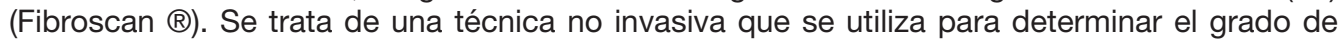
rigidez hepática, correlacionando el mismo al grado de fibrosis.

Las principales ventajas son que puede realizarse en forma ambulatoria, en pocos minutos, no es doloroso, la obtención de los resultados es inmediata, es operador independiente y reproducible tantas veces como se desee. ${ }^{(2)}$

En 2008 Lemoine y cols publican un estudio llevado a cabo entre enero 2004 y septiembre 2006 en pacientes con cirrosis hepática de etiología alcohólica o VHC, encontrando que la ET tenía una fuerte correlación con la HTP, definiendo la misma aquella superior a $10 \mathrm{mmHg} .^{(5)}$

En2011 Robic y cols demuestran que la ET no solo es efectiva en determinar la HTP, sino también en predecir la descompensación vinculada a la misma en pacientes con hepatopatía crónica. ${ }^{(6)}$

En 2013 Shi y cols publican un meta-análisis que incluyó 18 trabajos (3644 pacientes) que concluye que la ET es altamente precisa y útil en la detección de HTP significativa con una sensibilidad y especificidad del $90 \%$ y $79 \%$ respectivamente. ${ }^{(7)}$

Recientemente, Roccarina y cols realizaron una revisión en la que demuestran la existencia de una relación entre la resistencia hepática y el GPVH con un coeficiente de correlación de 0.783 , aunque éste disminuye para valores elevados de GPVH (>12mmHg). La buena sensibilidad (87\%) y especificidad (85\%) indican que el uso de la ET es bueno para diagnosticar HTP clínicamente significativa. ${ }^{(8)}$

Reiberger y cols evaluaron la relación entre la resistencia hepática y el GPVH en una cohorte de pacientes con cirrosis de distintas etiologías bajo tratamiento con beta-bloqueantes no selectivos, y describieron que esta relación mejoraba para un GPVH $>12 \mathrm{mmHg}$ en los "respondedores hemodinámicos".(8,9) 
En el Consenso Baveno VI, basándose en los resultados de estudios seleccionados por expertos, recomiendan una combinación de la medida de la resistencia hepática con el recuento plaquetario ( $>20 \mathrm{kPa}$ y $<150.000 / \mathrm{mm}^{3}$ plaquetas) para descartar la presencia de varices esofágicas que necesitan tratamiento en pacientes con enfermedad hepática crónica avanzada compensada. ${ }^{(10)}$

Con este algoritmo se evita realizar un $20 \%$ de las endoscopias totales. Siguiendo los criterios establecidos en el expanded-Baveno VI, en los cuales se agregaron otros dos estudios que fueron validados por expertos, tomando como valores de resistencia hepática $>25 \mathrm{kPa}$ y $<$ $110.000 / \mathrm{mm} 3$ plaquetas, se podrían evitar hasta un $40 \%$ de las endoscopias en los pacientes con enfermedad hepática crónica avanzada compensada ${ }^{(11)}$, aunque estos datos necesitan ser validados para algunas etiologías (enfermedad hepática colestásica, esteatosis hepática no alcohólica y cirrosis alcohólica).(8)

Hasta la fecha no se pueden descartar ni diagnosticar VEG de cualquier tamaño por métodos no invasivos con suficiente seguridad diagnóstica.

Con el objetivo de conocer la epidemiología de la cirrosis, correlacionar la presencia de HTP con los resultados de la elastografía de transición y los valores plaquetarios en la población de pacientes con cirrosis asistidos en la policlínica de hepatología del Hospital Pasteur, es que se realiza el presente trabajo.

\section{Material y Métodos}

Estudio observacional, descriptivo, retrospectivo y longitudinal en el periodo de 2015 a 2018.

Se consideró como población de estudio a los usuarios de ASSE, mayores de 18 años, de ambos sexos, que fueron asistidos y controlados en la policlínica de Hepatología del Hospital Pasteur con diagnóstico de cirrosis hepática de cualquier etiología, y que han sido valorados con ET realizado por el equipo médico de la policlínica.

Las variables que definieron el resultado del estudio fueron: presencia de HTP (valorado a través de la existencia de varices esofágicas, gastropatía por HTP, esplenomegalia y ascitis), valor de la rigidez hepática y recuento plaquetario.

Las variables a relevar fueron: edad, sexo y etiología de la cirrosis. Se consideraron como etiologías: infección por VHB, infección por VHC, consumo crónico de alcohol, enfermedad hepática autoinmune, hígado graso no alcohólico, y "otras", dentro de la cual se incluye cualquier causa que no haya sido contemplada en las demás variables.

El recuento plaquetario y la rigidez hepática fueron operacionalizadas definiendo los siguientes puntos de corte: $<150.000$ plaquetas $/ \mathrm{mm}^{3}$ y $\mathrm{ET}$ con $\mathrm{kPa} \geq 15$.

Para la descripción de las variables cuantitativas se calcularon media y desvío estándar; y en el caso de las variables categóricas, frecuencia absoluta y proporciones.

Se analizó la asociación de las variables categóricas o cualitativas mediante el test de Chi Cuadrado (X2) con corrección de Yates en los casos necesarios, y se calculó el odds ratio (OR). Se consideró como significativo un valor- $\mathrm{p}<0,05$.

Para el análisis de los datos se utilizó el software gratuito y de código abierto OpenEpi. (12)

Se obtuvieron los consentimientos informados de los participantes previo al acceso a las Historias Clínicas. Los datos se obtuvieron mediante revisión de dichas historias, siendo incluidas aquellas cuyos pacientes fueron diagnosticados con cirrosis hepática. Se utilizó una base de datos anonimizada creada en Microsoft Office Excel 2010, de acuerdo a las leyes vigentes en nuestro país (decreto 379/008), asegurando el derecho a la confidencialidad de los participantes.

Este proyecto de investigación obtuvo la aprobación del Comité de Ética de la Facultad de Medicina UdelaR el 26 de julio de 2018.

\section{Definiciones operacionales}

Várices esófago-gástricas: Son colaterales porto sistémicas, anastomosis profundas superiores. ${ }^{(13)}$ La presencia de varices esofagogástricas actualmente se evalúa mediante la realización de una fibrogastroscopía. 
Gastropatía por HTP: Trastornos circulatorios difusos de la mucosa gástrica, secundarios a la presencia de HTP. Pueden producir sangrados de diferente magnitud..$^{(1,14)}$

Ascitis: Es la presencia de líquido en la cavidad peritoneal, diagnosticada de forma clínica o por ecografía abdominal. ${ }^{(1)}$

Esplenomegalia: Crecimiento del tamaño del bazo, diagnosticado clínicamente o mediante ecografía, tomografía computada o resonancia nuclear magnética.

Plaquetopenia: recuento plaquetario $\leq 150.000 / \mathrm{mm}^{3}$.

Cirrosis: paciente con elementos clínicos - paraclínicos de insuficiencia hepatocítica, hipertensión portal, alteraciones anatomo-morfológicas del hígado (chico, nodular, heterogéneo), ET que evidencie rigidez hepática y/o confirmado por anatomía patológica que muestre fibrosis y nódulos de cualquier tamaño.

Alcoholismo: para que cause daño orgánico se considera un consumo semanal de $120 \mathrm{gr}$ etanol en el hombre o de $140 \mathrm{gr}$ etanol en la mujer por un período de dos o más años.

VHC: PCR positivo para VHC

VHB: pacientes con antígeno de superficie del VHB (HBsAg) positivo, anticuerpo anti core (Anti-HBc) total positivo mantenido por 6 meses.

Hígado graso no alcohólico: hallazgo imagenológico abdominal de esteatosis hepática, en ausencia de consumo de alcohol significativo o virus de hepatitis $B / C$, vinculado a la presencia de insulinoresistencia.

Hepatitis autoinmune: paciente con presencia de hepatitis autoinmune en etapa cirrótica según los criterios del Grupo Internacional de Hepatitis Autoinmune (GIHA).

\section{Resultados}

Se analizaron los datos de 49 pacientes con diagnóstico de cirrosis hepática, de los cuales 31 son de sexo masculino y 18 femenino.

En la distribución etaria de los participantes, las edades variaron entre 32 y 85 años, con una media de 56,6 \pm 12 años. Si se analiza la distribución de las edades diferenciando los pacientes por sexo, la edad media para el sexo masculino fue de 54,5 $\pm 11,2$ años, y para el sexo femenino $60,2 \pm 12,7$ años.

Las etiologías que se encontraron se observan en la figura 1. 
Figura 1: Etiologías halladas en la población estudiada $(n=49)$.

Tabla 1: Frecuencia de presencia de elementos de HTP $(n=34)$

${ }^{\star}$ ) Entre los 17 pacientes que presentaron más de un elemento clínico/paraclínico de HTP, encontramos que 12 asociaban 2, 4 asociaban 3 , y 1 asociaba los 4 elementos considerados en este estudio.

Tabla 2: Resultado de las asociaciones entre variables con la presencia de HTP

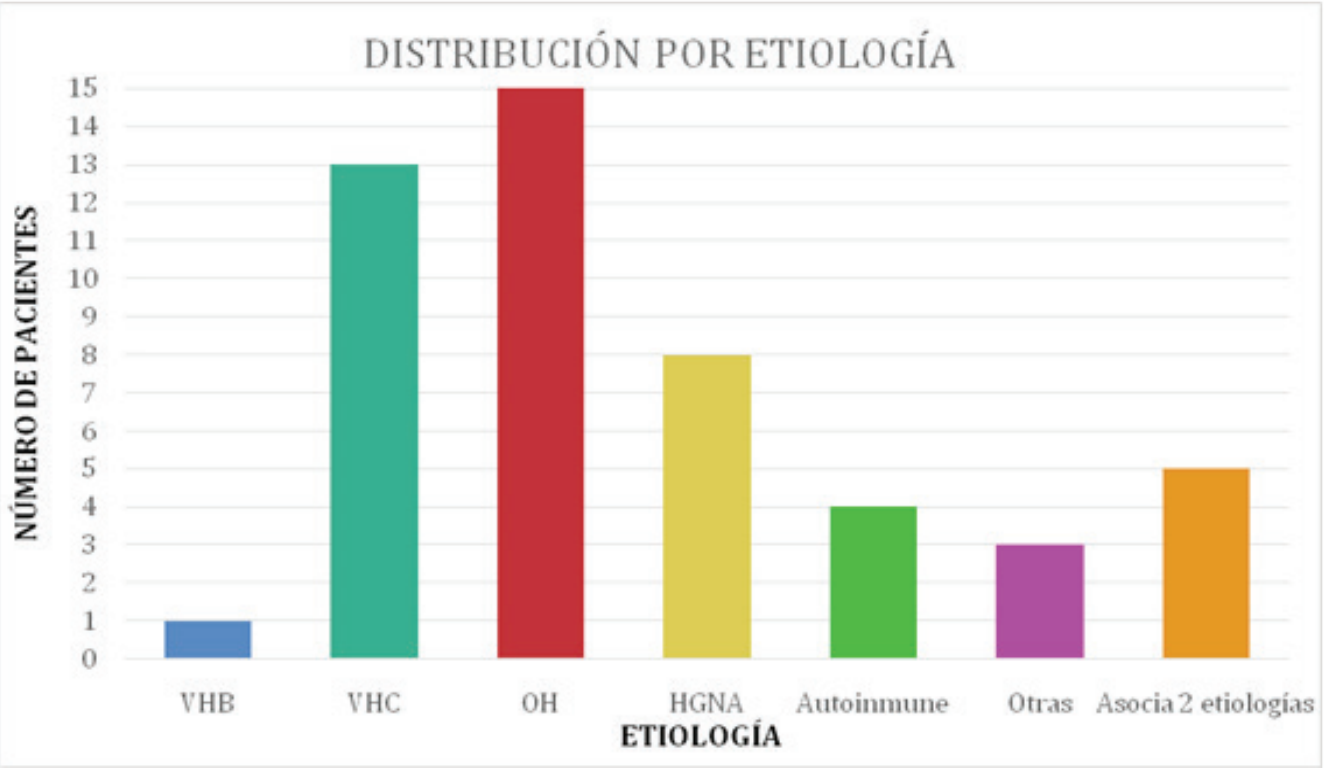

De los 49 pacientes estudiados, 34 presentaron elementos de HTP $(69,4 \%)$. En la Tabla 1 se muestra la frecuencia de las formas de presentación valoradas.

\begin{tabular}{|l|c|c|}
\hline \multicolumn{1}{|c|}{ ELEMENTO DE PRESENCIA DE HTP } & N & FRECUENCIA \\
\hline VEG & 6 & $17,6 \%$ \\
\hline GASTROPATÍA & 2 & $5,8 \%$ \\
\hline ESPLENOMEGALIA & 6 & $17,6 \%$ \\
\hline ASCITIS & 3 & $8,8 \%$ \\
\hline$>1$ ELEMENTO DE HTP $\left(^{*}\right)$ & 17 & $50,0 \%$ \\
\hline
\end{tabular}

Con respecto a los resultados de la ET, 32 de los 49 pacientes $(65,3 \%)$ tuvieron valores $\geq 15 \mathrm{kPa}$. En 29 pacientes $(59,2 \%)$ se encontraron valores de recuento plaquetario $\leq 150.000 /$ mm3. 23 pacientes $(46,9 \%)$ presentaron la combinación de ambas variables.

En la tabla 2 se muestran la asociación entre $\mathrm{kPa} \geq 15$ y Plaquetas $\leq 150.000 / \mathrm{mm}^{3}$ y la presencia de HTP.

\begin{tabular}{|l|l|l|l|l|}
\hline \multicolumn{1}{|c|}{ VARIABLE 1 } & VARIABLE 2 & \multicolumn{1}{c|}{ VALOR-P } \\
\hline $\mathrm{kPa} \geq 15$ & HTP & $\begin{array}{l}0,0052 \\
(\mathrm{~S})\end{array}$ & 7,71 & $(1,99 ; 29,99)$ \\
\hline Plaquetas $\leq 150.000$ & HTP & 0,24 & 2,09 & $(0,61 ; 7,19)$ \\
\hline $\begin{array}{l}\mathrm{kPa} \geq 15 \text { y Plaquetas } \leq \\
150.000\end{array}$ & HTP & $\begin{array}{l}0,028 \\
(\mathrm{~S})\end{array}$ & 5,71 & $(1,36 ; 24,06)$ \\
\hline
\end{tabular}

En la Tabla 3 se muestran la asociación entre $\mathrm{kPa} \geq 15$ y Plaquetas $\leq 150.000 / \mathrm{mm}^{3}$ y la presencia de várices esófago gástricas y/o gastropatía por hipertensión portal.

\begin{tabular}{|c|c|c|c|c|}
\hline VARIABLE 1 & VARIABLE 2 & VALOR-P & OR & IC \\
\hline $\mathrm{kPa} \geq 15$ & VEG & 0,16 & 2,53 & $(0,68 ; 9,47)$ \\
\hline $\begin{array}{c}\mathrm{kPa} \geq \begin{array}{c}15 \mathrm{y} \text { Plaquetas } \leq \\
150.000\end{array} \\
\text { VEG }\end{array}$ & 0,36 & 1,73 & $(0,54 ; 5,59)$ \\
\hline
\end{tabular}


Tabla 3: Resultado de las asociaciones entre variables con la presencia de VEG $\mathrm{y} / 0 \mathrm{GP}$

Tabla 4: Resultado de las asociaciones entre variables con la presencia de HTP en la población con etiologías más prevalente

\begin{tabular}{|c|c|c|c|c|}
\hline $\mathrm{kPa} \geq 15$ & VEG y/o GP & 0,32 & 1,83 & $(0,55 ; 6,16)$ \\
\hline \begin{tabular}{c}
$\mathrm{kPa} \geq \begin{array}{c}15 \text { y Plaquetas } \leq \\
150.000\end{array}$ \\
\hline
\end{tabular} & VEG y/o GP & 0,85 & 0,89 & $(0,29 ; 2,78)$ \\
\hline
\end{tabular}

En la Tabla 4 se muestran la asociación entre $\mathrm{kPa} \geq 15$ y Plaquetas $\leq 150.000 / \mathrm{mm}$ y la presencia de HTP para los pacientes con cirrosis de etiología alcohólica y VHC.

\begin{tabular}{|c|c|c|}
\hline VARIABLE 1 & HTP & $0,037 \quad$ (S) \\
\hline $\mathrm{kPa} \geq 15$ para etiología $\mathrm{OH}$ & HTP & \multicolumn{2}{c|}{0,28} \\
\hline $\mathrm{kPa} \geq 15$ y Plaquetas $\leq 150.000$ para etiología OH & HTP & $0,039 \quad(\mathrm{~S})$ \\
\hline $\mathrm{kPa} \geq 15$ para etiología VHC & HTP & $0,039 \quad$ (S) \\
\hline $\mathrm{kPa} \geq 15$ y Plaquetas $\leq 150.000$ para etiología VHC & HTRLE \\
\hline
\end{tabular}

\section{Discusión}

El estudio incluyó un total de 49 pacientes, predominando el sexo masculino (32/18). La edad media de la muestra fue de 56,6 \pm 12 años y las etiologías que prevalecieron fueron la cirrosis alcohólica (15/49) y la infección por VHC (13/49) al igual a lo observado a nivel internacional.

La prevalencia de la etiología alcohólica se relaciona a la gran prevalencia del consumo de alcohol en nuestro país. Según los datos que arroja el censo del año 2008 de la Junta Nacional de Drogas, un $50.1 \%$ de la población consume habitualmente alcohol y un $9.3 \%$ de los mismos (75.000 personas) muestran signos de dependencia. Dentro de los consumidores habituales el $60.2 \%$ son de sexo masculino ${ }^{(15)}$

El consumo crónico de alcohol es considerado uno de los problemas sanitarios actuales del país y lograr su descenso es una meta sanitaria nacional para el año 2020. Para lograr tal objetivo se han planteado estrategias nacionales que apuntan a disminuir la accesibilidad y disponibilidad de las bebidas alcohólicas, reducir los daños ocasionados por los siniestros de tránsito relacionados con el consumo de alcohol y regularizar su publicidad y venta. ${ }^{(16)}$

Aunque en nuestro país no existen datos epidemiológicos sobre la prevalencia de VHC, explorando datos de la región y del banco de sangre se estima que en Uruguay hay un 1,5\% de la población portadora de VHC (52.000 personas). Ya que es una enfermedad que en la mayoría de casos no genera síntomas tempranos, hay un alto porcentaje de pacientes portadores de VHC que no lo saben y son diagnosticados en el contexto de una donación o por un hepatograma anormal, por lo que existe alta prevalencia de cirrosis hepática al momento del diagnóstico. ${ }^{(17)}$

Del análisis se desprende que 32 pacientes de los 49 estudiados presentaban HTP, siendo esto esperable dada las características de la población estudiada. La HTP tiene diversas formas de presentación clínica y dentro del estudio realizado el $50 \%$ de los pacientes se manifestaron con más de una. Esto determina la necesidad de su búsqueda no sólo a través del examen físico completo y dirigido a la misma, sino también mediante la realización de una fibrogastroscopía y ecografía doppler abdominal.

Encontramos una asociación estadísticamente significativa entre la presencia de valores de ET $\geq 15 \mathrm{kPa}$ y la presencia de HTP, por lo que el punto de corte escogido fue adecuado. Hecho similar a los hallazgos de estudios internacionales. ${ }^{(5,6,8)}$

El análisis de la variable plaquetopenia $\leq 150.000 / \mathrm{mm} 3$ y su vinculación con HTP no demostró asociación. Una de las consideraciones que debemos realizar es si el punto de corte fue el adecuado; los estudios internacionales son variables en los mismos, el consenso Baveno IV utiliza $\leq 110.000 / \mathrm{mm} 3$.

Aunque el recuento plaquetario bajo se indica como un método seguro para diagnosticar HTP y VEG, un estudio reciente multicéntrico del Portal Hypertension Collaborative Group llegó a la conclusión de que un corte seccional del conteo de plaquetas es inadecuado; por lo que debemos preguntarnos si nuestros resultados serían los mismos si hubiésemos considerado un promedio del valor de plaquetas de los pacientes en un período de tiempo determinado, 
considerando que el recuento plaquetario de estos pacientes tiene una oscilación mayor que la población no cirrótica. ${ }^{(18)}$

Demostramos una asociación significativa cuando analizamos las dos variables juntas (ET con $\mathrm{kPa} \geq 15$ y Plaquetas $\leq 150.000 / \mathrm{mm} 3$ ) vinculadas a la presencia de HTP. Seguramente esto se deba a la muy importante asociación presente con la ET y la HTP ya analizada.

Analizando nuestras variables de análisis ( $E T$ con $\mathrm{kPa} \geq 15$ y Plaquetas $/ \mathrm{mm} 3 \leq 150.000$ ) y los hallazgos de la fibrogastroscopía (VEG/VEG y/o GP) no encontramos asociación estadísticamente significativa. Debemos recordar que la fibrogastroscopía es un estudio técnico dependiente y que no ha sido realizada en todos los pacientes por el mismo técnico, lo que puede conducir a distintas interpretaciones sobre todo en la presencia de gastropatía de la hipertensión portal, ya que requiere de la experiencia del médico endoscopista para su reconocimiento. Otro elemento que puede influir en estos hallazgos es el número de casos analizados; los estudios internacionales que analizaron estas variables reportaban varios cientos de pacientes.

La fibrogastroscopía es un estudio invasivo, no siempre aceptado por el paciente; pero en virtud de los hallazgos de nuestra población, debemos continuar planteando su realización para el diagnóstico de VEG y/o GP en nuestros pacientes.

Cuando vinculamos la etiología alcohólica y VHC con HTP según la variable ET con $\mathrm{kPa} \geq$ 15 encontramos una asociación significativa en ambos casos. No fue así cuando buscamos la asociación usando las variables ET con $\mathrm{kPa} \geq 15$ y Plaquetas $\leq 150.000 / \mathrm{mm}^{3}$ y la presencia de HTP, no existiendo asociación significativa para la población con etiología alcohólica, pero sí en los que presentan etiología por VHC.

Por lo tanto, la ET con valores de $\mathrm{kPa} \geq 15$ no sólo es útil para el diagnóstico de HTP en la población de cirróticos en general, sino que es útil para las etiologías analizadas que son las más prevalentes en nuestra población.

\section{Conclusiones y Perspectivas}

Mayor prevalencia de cirrosis en hombres con etiología alcohólica y VHC

Alta prevalencia HTP en cirrosis.

Existe asociación de HTP con ET $\geq 15 \mathrm{kPa}$

Existe asociación de HTP con ET $\geq 15 \mathrm{kPa}+$ plaquetas $<150.000 / \mathrm{mm}^{3}$

Existe asociación de HTP con ET $\geq 15 \mathrm{kPa}$ en etiología alcohólica y VHC

Existe asociación de HTP con ET $\geq 15 \mathrm{kPa}$ + plaquetas $<150.000 / \mathrm{mm}^{3}$ en etiología VHC.

No fue posible demostrar la asociación de la presencia de VEG y/o GP con valores de ET $\geq$ $15 \mathrm{kPa}$ por lo que se mantiene la recomienda de continuar realizando la fibrogastroscopia para el diagnóstico de HTP en nuestros pacientes.

\section{Bibliografía}

1- Rozman Borstnar C, Cardellach F. Hipertensión portal y enfermedades vasculares del hígado. En: Farreras Rozman. Medicina interna. 18 ed. Barcelona: Elsevier; 2016; p. 264-276.

2- Pareja Q JS, Restrepo G JC. Diagnostic Methods in Portal Hypertension. Revcolombgastroenterol [Internet]. 2016 [acceso: 15/05/2019];31(2):135-45. Disponible en: http://www.scielo.org.co/scielo. php?script=sci_arttext\&pid=S0120-99572016000200006

3- Zeballos E, Cohen H, Taullard D. Semiología gastroenterológica. Montevideo: Oficina del Libro FEFMUR; 2003

4- Garcia-Tsao G, Sanyal AJ, Grace ND, Carey W, Shuhart MC, Davis GL, et al. Prevention and management of gastroesophagealvarices and variceal hemorrhage in cirrhosis. VolHepatology. 2007 Sep[acceso: 15/05/2018];46(3):922-38. Disponible en: https://gi.org/guideline/prevention-andmanagement-of-gastroesophageal-varices-and-variceal-hemorrhage-in-cirrhosis/

5- Lemoine M, Katsahian S, Ziol M, Nahon P, Ganne-Carrie N, Kazemi F, et al. Liver stiffness measurement as a predictive tool of clinically significant portal hypertension in patients with compensated hepatitis $\mathrm{C}$ virus or alcohol-related cirrhosis. Aliment PharmacolTher. 2008;28(9):1102-10. 
6- Robic MA, Procopet B, Métivier S, Péron JM, Selves J, Vinel JP, et al. Liver stiffness accurately predicts portal hypertension related complications in patients with chronic liver disease: a prospective study. $J$ Hepatol. 2011;55(5):1017-24.

7- Shi KQ, Fan YC, Pan ZZ, Lin XF, Liu WY, Chen YP, et al. Transient elastography: a meta-analysis of diagnostic accuracy in evaluation of portal hypertension in chronic liver disease. Liver Int. 2013;33(1):62-71.

8- Roccarina D, Rosselli M, Genesca J, Tsochatzis EA. Elastography methods for the non-invasive assessment of portal hypertension. Expert Rev GastroenterolHepatol. 2018;12(2):155-64.

9- Reiberger T, Ferlitsch A, Payer BA, Pinter M, Homoncik M, Peck-Radosavljevic M. Nonselective B-blockers improve the correlation of liver stiffness and portal pressure in advanced cirrhosis. J Gastroenterol. 2012; 47(5):561-8.

10- De Franchis R, Abraldes JG, Bajaj J, Berzigotti A, Bosch J, Burroughs AK, et al. Expanding consensus in portal hypertension Report of the Baveno VI Consensus Workshop: Stratifying risk and individualizing care for portal hypertension. J Hepatol. 2015;743-52.

11- Augustin S, Pons M, Maurice JB, Bureau C, Stefanescu H, Ney M, et al. Expanding the Baveno VI criteria for the screening of varices in patients with compensated advanced chronic liver disease. Hepatology. 2017;66(6):1980-8.

12- Dean AG, Sullivan KM, Soe MM. OpenEpi: Open Source Epidemiologic Statistics for Public Health[base de datos en Internet],Atlanta: Open Epi; 2007[actualizado 2013/04/06, acceso 15/05/2018]. Disponible en: https://www.openepi.com

13- Colombato L, Bandi JC, Mendizabal M, Paz S, Súarez S, Villaverde J. Guías de manejo de las varices esófago-gástricas. AAEEH. 2015;(1004):1-8.

14- Guillén Isern D, Subirat Esquivel L. Consideraciones actuales de la gastropatía hipertensiva portal. Rev Arch Médico Camagüey. 2011;15(3):626-37.

15- Uruguay. Junta Nacional de Drogas. Drogas: más información, menos riesgos. $6^{\circ}$ ed. Montevideo: IMPO; 2007.

16- Uruguay. Ministerio de Salud Pública. Objetivos Sanitarios Nacionales 2020 [Internet]. Montevideo: MSP; 2016 [acceso: 04/09/2018]. Disponible en: http://siteanii.anii.org.uy/upcms/files/objetivossanitarios-nacionales-2020.pdf

17- Hernández, N. Situación del VHC en Uruguay [Internet]. Montevideo: Clínica de Gastroenterología; 2014 [acceso: 04/09/2018]. Disponible en: http://server241.dinamichosting.com/gastro/wp-content/ uploads/2015/12/3_ppt_ProfNHernandez.pdf

18- Qamar AA, Grace ND, Groszmann RJ, Garcia-tsao G, Bosch J, Burroughs AK, et al. Platelet Count Is Not a Predictor of the Presence or Development of GastroesophagealVarices in Cirrhosis. [Internet]. AASLD. 2008[acceso: 15/05/2018];47(1):153-9. aasldpubs.onlinelibrary.wiley.com/doi/full/10.1002/hep.21941. Disponible en: https://

\section{Aporte cada autor al trabajo}

Laura Perez: concepción y diseño del trabajo, recolección de datos, análisis e interpretación de datos, redacción del manuscrito.

Valentina Pincelli: concepción y diseño del trabajo, recolección de datos, análisis e interpretación de datos, redacción del manuscrito.

Lorena Pittamiglio: concepción y diseño del trabajo, recolección de datos, análisis e interpretación de datos, redacción del manuscrito.

Mateo Rodríguez Pezzino: concepción y diseño del trabajo, recolección de datos, análisis e interpretación de datos, redacción del manuscrito.

Damián Santos: concepción y diseño del trabajo, recolección de datos, análisis e interpretación de datos, redacción del manuscrito.

Micaela Saralegui: concepción y diseño del trabajo, recolección de datos, análisis e interpretación de datos, redacción del manuscrito.

Gastón Nieto: concepción y diseño del trabajo, análisis e interpretación de los datos o resultados, redacción del manuscrito.

Mercedes Perendones: concepción y diseño del trabajo, análisis e interpretación de los datos, análisis estadístico de los datos, redacción del manuscrito, revisión crítica del manuscrito.

Marcelo Chiarella: redacción del manuscrito, revisión crítica del manuscrito. 